\title{
ON THE CONVOLUTION OF HETEROGENEOUS BERNOULLI RANDOM VARIABLES
}

\author{
MAOCHAO XU, ${ }^{*}$ Illinois State University \\ N. BALAKRISHNAN, ${ }^{* *}$ McMaster University
}

\begin{abstract}
In this paper, some ordering properties of convolutions of heterogeneous Bernoulli random variables are discussed. It is shown that, under some suitable conditions, the likelihood ratio order and the reversed hazard rate order hold between convolutions of two heterogeneous Bernoulli sequences. The results established here extend and strengthen the previous results of Pledger and Proschan (1971) and Boland, Singh and Cukic (2002).
\end{abstract}

Keywords: Bernoulli; heterogeneous variable; likelihood ratio order; majorization; reversed hazard rate order

2010 Mathematics Subject Classification: Primary 60E15; 62N05; 62G30; 62D05

\section{Introduction}

The Bernoulli distribution is one of the most fundamental distributions in statistics, and has found key applications in engineering, actuarial science, operations research, and reliability theory. Recently, much attention has been paid to the study of convolutions of independent random variables, such as exponential, gamma, Weibull, and geometric. We refer the reader to Zhao and Balakrishnan (2010), Mao et al. (2010), Kochar and Xu (2011), and the references therein for some related developments.

Let $X_{p_{1}}, \ldots, X_{p_{n}}$ be a sequence of independent Bernoulli random variables with parameters $p_{1}, \ldots, p_{n}$, respectively. The convolution of independent Bernoulli random variables was first considered in Hoeffding (1956), who showed that

$$
\mathrm{P}\left(\sum_{i=1}^{n} X_{p_{i}} \leq k\right) \leq \sum_{j=0}^{k}\left(\begin{array}{l}
n \\
j
\end{array}\right) \bar{p}^{j}(1-\bar{p})^{n-j} \quad \text { for } 0 \leq k \leq n \bar{p}-1
$$

and

$$
\mathrm{P}\left(\sum_{i=1}^{n} X_{p_{i}} \leq k\right) \geq \sum_{j=0}^{k}\left(\begin{array}{l}
n \\
j
\end{array}\right) \bar{p}^{j}(1-\bar{p})^{n-j} \quad \text { for } n \bar{p} \leq k \leq n,
$$

where $\bar{p}=\sum_{i=1}^{n} p_{i} / n$. As a consequence, Hoeffding further showed that, for any two integers $b$ and $c$ such that $0 \leq b \leq n \bar{p} \leq c \leq n$,

$$
\mathrm{P}\left(b \leq \sum_{i=1}^{n} X_{p_{i}} \leq c\right) \geq \sum_{j=b}^{c}\left(\begin{array}{l}
n \\
j
\end{array}\right) \bar{p}^{j}(1-\bar{p})^{n-j} .
$$

Received 14 September 2010; revision received 24 February 2011.

* Postal address: Department of Mathematics, Illinois State University, Normal, IL, USA.

Email address: mxu2@ilstu.edu

** Postal address: Department of Mathematics and Statistics, McMaster University, Hamilton, Ontario, Canada. 
Gleser (1975), by utilizing the concept of majorization, refined the results of Hoeffding (1956) and showed that if

$$
\left(p_{1}, \ldots, p_{n}\right) \succeq_{\mathrm{m}}\left(p_{1}^{*}, \ldots, p_{n}^{*}\right)
$$

then

$$
\mathrm{P}\left(\sum_{i=1}^{n} X_{p_{i}} \leq k\right) \leq \mathrm{P}\left(\sum_{i=1}^{n} X_{p_{i}^{*}} \leq k\right) \text { for } 0 \leq k \leq n \bar{p}-2
$$

and

$$
\mathrm{P}\left(\sum_{i=1}^{n} X_{p_{i}} \leq k\right) \geq \mathrm{P}\left(\sum_{i=1}^{n} X_{p_{i}^{*}} \leq k\right) \text { for } n \bar{p}+2 \leq k \leq n,
$$

where ' $\succeq_{\mathrm{m}}$ ' denotes the majorization order (see Definition 2.2 below).

This result was further extended in Boland and Proschan (1983), who proved that, under condition (1.1), if $p_{i} \geq k /(n-1)$ for all $i=1, \ldots, n$ then

$$
\mathrm{P}\left(\sum_{i=1}^{n} X_{p_{i}} \leq k\right) \leq \mathrm{P}\left(\sum_{i=1}^{n} X_{p_{i}^{*}} \leq k\right)
$$

and if $p_{i} \leq k /(n-1)$ for all $i=1, \ldots, n$ then

$$
\mathrm{P}\left(\sum_{i=1}^{n} X_{p_{i}} \leq k\right) \geq \mathrm{P}\left(\sum_{i=1}^{n} X_{p_{i}^{*}} \leq k\right)
$$

It is also of interest to mention that Pledger and Proschan (1971) established the following results:

$$
\left(-\log \left(p_{1}\right), \ldots,-\log \left(p_{n}\right)\right) \succeq_{\mathrm{m}}\left(-\log \left(p_{1}^{*}\right), \ldots,-\log \left(p_{n}^{*}\right)\right) \Longrightarrow \sum_{i=1}^{n} X_{p_{i}} \geq_{\mathrm{st}} \sum_{i=1}^{n} X_{p_{i}^{*}}
$$

and

$$
\left(\frac{1-p_{1}}{p_{1}}, \ldots, \frac{1-p_{n}}{p_{n}}\right) \succeq_{\mathrm{m}}\left(\frac{1-p_{1}^{*}}{p_{1}^{*}}, \ldots, \frac{1-p_{n}^{*}}{p_{n}^{*}}\right) \Longrightarrow \sum_{i=1}^{n} X_{p_{i}} \geq_{\mathrm{st}} \sum_{i=1}^{n} X_{p_{i}^{*}},
$$

where ' $\geq_{\text {st }}$ ' denotes the usual stochastic order (see Definition 2.1 below).

Wang (1993) considered the variance of the convolution of independent Bernoulli random variables, and proved that

$$
\left(p_{1}, \ldots, p_{n}\right) \succeq_{\mathrm{m}}\left(p_{1}^{*}, \ldots, p_{n}^{*}\right) \Longrightarrow \operatorname{var}\left(\sum_{i=1}^{n} X_{p_{i}}\right) \leq \operatorname{var}\left(\sum_{i=1}^{n} X_{p_{i}^{*}}\right)
$$

which means that the variance of the convolution increases as the components of $\left(p_{1}, \ldots, p_{n}\right)$ become more homogeneous. Actually, a stronger version of this result has been proved in Karlin and Novikoff (1963) by using the convex transforms; see also Ma (2000) and Hu and Ruan (2004) for some additional results in this direction.

More recently, Boland et al. (2002), (2004) further studied the comparison of convolutions of heterogeneous and homogeneous Bernoulli random variables. Specifically, letting $Y_{1}, \ldots, Y_{n}$ 
be independent and identically distributed Bernoulli random variables with parameter $p$, they showed that

$$
\sum_{i=1}^{n} X_{p_{i}} \geq_{\mathrm{st}} \sum_{i=1}^{n} Y_{i} \Longleftrightarrow p \leq p_{\mathrm{g}}
$$

and

$$
\sum_{i=1}^{n} X_{p_{i}} \leq_{\mathrm{st}} \sum_{i=1}^{n} Y_{i} \Longleftrightarrow p \geq p_{\mathrm{cg}}
$$

where $p_{\mathrm{g}}=\sqrt[n]{\prod_{i=1}^{n} p_{i}}$ and $p_{\mathrm{cg}}=1-\sqrt[n]{\prod_{i=1}^{n}\left(1-p_{i}\right)}$ are the geometric mean of $p_{i}$ and the complement of the geometric mean of $1-p_{i}$, respectively. Furthermore,

$$
\sum_{i=1}^{n} X_{p_{i}} \geq \mathrm{hr} \sum_{i=1}^{n} Y_{i} \Longleftrightarrow \sum_{i=1}^{n} X_{p_{i}} \geq_{\operatorname{lr}} \sum_{i=1}^{n} Y_{i} \quad \Longleftrightarrow \quad p \leq p_{\mathrm{h}}
$$

and

$$
\sum_{i=1}^{n} X_{p_{i}} \leq \mathrm{hr} \sum_{i=1}^{n} Y_{i} \Longleftrightarrow \sum_{i=1}^{n} X_{p_{i}} \leq \mathrm{lr} \sum_{i=1}^{n} Y_{i} \Longleftrightarrow p \geq p_{\mathrm{ch}},
$$

where $p_{\mathrm{h}}=n / \sum_{i=1}^{n} 1 / p_{i}$ and $p_{\mathrm{ch}}=1-n / \sum_{i=1}^{n} 1 /\left(1-p_{i}\right)$ are the harmonic mean of $p_{i}$ and the complement of the harmonic mean of $1-p_{i}$, respectively, and ' $\geq_{\mathrm{hr}}$ ' and ' $\geq_{\mathrm{lr}}$ ' denote the hazard rate order and the likelihood ratio order, respectively (see Definition 2.1 below). We refer the reader to Boland and Singh (2006) and Boland (2007) for reviews of various developments on this topic.

In this paper we continue further in this direction and show that

$$
\left(-\log \left(p_{1}\right), \ldots,-\log \left(p_{n}\right)\right) \stackrel{\mathrm{w}}{\succeq}\left(-\log \left(p_{1}^{*}\right), \ldots,-\log \left(p_{n}^{*}\right)\right) \Longrightarrow \sum_{i=1}^{n} X_{p_{i}} \geq_{\mathrm{rh}} \sum_{i=1}^{n} X_{p_{i}^{*}}
$$

and

$$
\left(\frac{1}{p_{1}}, \ldots, \frac{1}{p_{n}}\right) \stackrel{\mathrm{w}}{\succeq}\left(\frac{1}{p_{1}^{*}}, \ldots, \frac{1}{p_{n}^{*}}\right) \Longrightarrow \sum_{i=1}^{n} X_{p_{i}} \geq \operatorname{lr} \sum_{i=1}^{n} X_{p_{i}^{*}},
$$

or, equivalently,

$$
\left(\frac{1-p_{1}}{p_{1}}, \ldots, \frac{1-p_{n}}{p_{n}}\right) \stackrel{\mathrm{w}}{\succeq}\left(\frac{1-p_{1}^{*}}{p_{1}^{*}}, \ldots, \frac{1-p_{n}^{*}}{p_{n}^{*}}\right) \Longrightarrow \sum_{i=1}^{n} X_{p_{i}} \geq_{\operatorname{lr}} \sum_{i=1}^{n} X_{p_{i}^{*}},
$$

where ' $\succeq$ ' denotes the weak supermajorization order (see Definition 2.3 below), and ' $\geq_{\mathrm{rh}}$ ' denotes the reversed hazard rate order (see Definition 2.1 below). Our results not only strengthen Equations (1.2) and (1.3) of Pledger and Proschan (1971), but also generalize the results of Boland et al. (2002) from homogeneous to heterogeneous Bernoulli random variables. It should also be pointed out that the condition in (1.5) is stronger than that in (1.4) (see Marshall and Olkin (1979, p. 117)). Some applications of these results in reliability theory and software testing can be found in Pledger and Proschan (1971), Boland et al. (2002), and Xu and Balakrishnan (2010). 


\section{Preliminaries}

In this section we first recall some notions of stochastic orders and majorization orders which are closely related to the main results to be developed in the following section.

Definition 2.1. Let $X$ and $Y$ be two discrete random variables with common support on the integers $\mathbb{N}_{0}=\{0,1,2, \ldots\}$, respective probability mass functions $f(k)$ and $g(k)$, respective distribution functions $F(k)=\mathrm{P}(X \leq k)$ and $G(k)=\mathrm{P}(Y \leq k)$, and respective survival functions $\bar{F}(k)=\mathrm{P}(X \geq k)$ and $\bar{G}(k)=\mathrm{P}(Y \geq k)$. Then, $X$ is said to be smaller than $Y$ in the

(i) likelihood ratio order, denoted by $X \leq 1 \mathrm{r} Y$, if $g(k) / f(k)$ is increasing in $k \in \mathbb{N}_{0}$;

(ii) hazard rate order, denoted by $X \leq_{\mathrm{hr}} Y$, if $\bar{G}(k) / \bar{F}(k)$ is increasing in $k \in \mathbb{N}_{0}$;

(iii) reversed hazard rate order, denoted by $X \leq_{\mathrm{rh}} Y$, if $G(k) / F(k)$ is increasing in $k \in \mathbb{N}_{0}$;

(iv) usual stochastic order, denoted by $X \leq_{\text {st }} Y$, if $\bar{F}(k) \leq \bar{G}(k)$ for all $k \in \mathbb{N}_{0}$.

The following implications are well known:

$$
X \leq_{\mathrm{lr}} Y \quad \Longrightarrow \quad X \leq_{\mathrm{hr}(\mathrm{rh})} Y \quad \Longrightarrow \quad X \leq_{\mathrm{st}} Y
$$

For a comprehensive discussion on various stochastic orders, we refer the reader to Shaked and Shanthikumar (2007).

We shall also use the concept of majorization in the development of our main results. Let $\left\{x_{(1)}, x_{(2)}, \ldots, x_{(n)}\right\}$ denote the increasing arrangement of the components of the vector $\boldsymbol{x}=$ $\left(x_{1}, x_{2}, \ldots, x_{n}\right)$.

Definition 2.2. The vector $\boldsymbol{y}$ is said to be majorized by the vector $\boldsymbol{x}$ (denoted by $\boldsymbol{x} \succeq_{\mathrm{m}} \boldsymbol{y}$ ) if

$$
\sum_{i=1}^{j} x_{(i)} \leq \sum_{i=1}^{j} y_{(i)}
$$

for $j=1, \ldots, n-1$ and $\sum_{i=1}^{n} x_{(i)}=\sum_{i=1}^{n} y_{(i)}$.

By relaxing the last equality condition we have the following definition.

Definition 2.3. The vector $\boldsymbol{y}$ is said to be weakly supermajorized by vector $\boldsymbol{x}$ (denoted by $\boldsymbol{x} \succeq \boldsymbol{\mathrm { w }}$ ) if

$$
\sum_{i=1}^{j} x_{(i)} \leq \sum_{i=1}^{j} y_{(i)}
$$

for $j=1, \ldots, n$.

For extensive and comprehensive details on the theory of majorization orders and their applications, we refer the reader to the book by Marshall and Olkin (1979).

\section{Main results}

The following lemma will be used in the sequel (see Theorem A.8 of Marshall and Olkin (1979, p. 59)). 
Lemma 3.1. A real-valued function $\phi$ defined on $\mathbb{R}^{n}$ satisfies

$$
\boldsymbol{x} \stackrel{\mathrm{W}}{\geq} \boldsymbol{y} \Longrightarrow \phi(x) \geq \phi(y)
$$

if and only if $\phi$ is decreasing and Schur convex on $\mathbb{R}^{n}$.

Let us first discuss the two-dimensional case.

Lemma 3.2. Let $X_{p_{1}}$ and $X_{p_{2}}$ be independent Bernoulli random variables with parameters $p_{1}$ and $p_{2}$. Then,

$$
\left(\frac{1}{p_{1}}, \frac{1}{p_{2}}\right) \succeq\left(\frac{1}{p_{1}^{*}}, \frac{1}{p_{2}^{*}}\right) \Longrightarrow X_{p_{1}}+X_{p_{2}} \geq \operatorname{lr} X_{p_{1}^{*}}+X_{p_{2}^{*}} .
$$

Proof. Define, for $j=0,1,2$,

$$
f(j)=\mathrm{P}\left(X_{p_{1}}+X_{p_{2}}=j\right)
$$

and

$$
g(j)=\mathrm{P}\left(X_{p_{1}^{*}}+X_{p_{2}^{*}}=j\right) .
$$

Note that $\left(1 / p_{1}, 1 / p_{2}\right) \stackrel{\mathrm{w}}{\succeq}\left(1 / p_{1}^{*}, 1 / p_{2}^{*}\right)$ implies that

$$
\frac{1}{p_{1}}+\frac{1}{p_{2}} \leq \frac{1}{p_{1}^{*}}+\frac{1}{p_{2}^{*}}
$$

Now, let us show that $X_{p_{1}}+X_{p_{2}} \geq_{\operatorname{lr}} X_{p_{1}^{*}}+X_{p_{2}^{*}}$. This is true if and only if $f(j) / g(j)$ is increasing in $j=0,1,2$, or, equivalently,

$$
\frac{\left(1-p_{1}\right)\left(1-p_{2}\right)}{\left(1-p_{1}^{*}\right)\left(1-p_{2}^{*}\right)} \leq \frac{p_{1}+p_{2}-2 p_{1} p_{2}}{p_{1}^{*}+p_{2}^{*}-2 p_{1}^{*} p_{2}^{*}} \leq \frac{p_{1} p_{2}}{p_{1}^{*} p_{2}^{*}} .
$$

The second inequality of (3.2) follows from (3.1). Hence, it is enough for us to show the first inequality, which could be simplified as

$$
\frac{1}{1 / p_{1}^{*}-1}+\frac{1}{1 / p_{2}^{*}-1} \leq \frac{1}{1 / p_{1}-1}+\frac{1}{1 / p_{2}-1} .
$$

Observing that

$$
h(x, y)=\frac{1}{x-1}+\frac{1}{y-1}, \quad x>1, y>1,
$$

is the sum of decreasing convex functions, $h(x, y)$ is decreasing Schur convex (see Theorem C. 1 of Marshall and Olkin, (1979, p. 64)). Hence, upon using Lemma 3.1, (3.3) follows immediately.

Lemma 3.3. Let $X_{p_{1}}$ and $X_{p_{2}}$ be independent Bernoulli random variables with parameters $p_{1}$ and $p_{2}$. Then,

$$
\left(-\log \left(p_{1}\right),-\log \left(p_{2}\right)\right) \stackrel{\mathrm{w}}{\geq}\left(-\log \left(p_{1}^{*}\right),-\log \left(p_{2}^{*}\right)\right) \quad \Longrightarrow \quad X_{p_{1}}+X_{p_{2}} \geq_{\mathrm{rh}} X_{p_{1}^{*}}+X_{p_{2}^{*}}
$$


Proof. Define, for $j=0,1,2$,

$$
F(j)=\mathrm{P}\left(X_{p_{1}}+X_{p_{2}} \leq j\right)
$$

and

$$
G(j)=\mathrm{P}\left(X_{p_{1}^{*}}+X_{p_{2}^{*}} \leq j\right) .
$$

Note that $\left(-\log \left(p_{1}\right),-\log \left(p_{2}\right)\right) \stackrel{\mathrm{W}}{\succeq}\left(-\log \left(p_{1}^{*}\right),-\log \left(p_{2}^{*}\right)\right)$ implies that

$$
p_{1} p_{2} \geq p_{1}^{*} p_{2}^{*} \text {. }
$$

We now have to show that

$$
\frac{F(j)}{G(j)}
$$

is increasing in $j=0,1,2$ for the reversed hazard rate order, which is equivalent to showing that

$$
\frac{\left(1-p_{1}\right)\left(1-p_{2}\right)}{\left(1-p_{1}^{*}\right)\left(1-p_{2}^{*}\right)} \leq \frac{1-p_{1} p_{2}}{1-p_{1}^{*} p_{2}^{*}} \leq 1
$$

The second inequality of (3.5) follows from (3.4). Now, let us show the first inequality, which could be simplified as

$$
\frac{1}{1 / p_{1}^{*}-1}+\frac{1}{1 / p_{2}^{*}-1} \leq \frac{1}{1 / p_{1}-1}+\frac{1}{1 / p_{2}-1}
$$

Since

$$
h_{1}(x, y)=\frac{1}{\mathrm{e}^{x}-1}+\frac{1}{\mathrm{e}^{y}-1}, \quad x>0, y>0,
$$

is the sum of decreasing convex functions, $h_{1}(x, y)$ is decreasing Schur convex. Hence, by using Lemma 3.1 once again, (3.5) follows immediately.

The following two results present natural extensions of Lemmas 3.2 and 3.3 from the twodimensional case to the general case. The technique used here is similar to that used in Bon and Păltănea (1999).

Theorem 3.1. Let $X_{p_{1}}, \ldots, X_{p_{n}}$ be independent Bernoulli random variables with parameters $p_{1}, \ldots, p_{n}$, respectively. Then,

$$
\left(\frac{1}{p_{1}}, \ldots, \frac{1}{p_{n}}\right) \stackrel{\mathrm{w}}{\succeq}\left(\frac{1}{p_{1}^{*}}, \ldots, \frac{1}{p_{n}^{*}}\right) \Longrightarrow X_{p_{1}}+\cdots+X_{p_{n}} \geq_{\mathrm{rr}} X_{p_{1}^{*}}+\cdots+X_{p_{n}^{*}} .
$$

Proof. Without loss of generality, assume that $p_{1} \leq p_{2} \leq \cdots \leq p_{n}$ and $p_{1}^{*} \leq p_{2}^{*} \leq \cdots \leq$ $p_{n}^{*}$. The proof is carried out by mathematical induction. For $n=1$, the proof is trivial. When $n=2$, the result follows from Lemma 3.2. Now, assume that the result holds for $n-1$. We will then prove the case for $n \geq 3$. Note that

$$
\left(\frac{1}{p_{1}}, \ldots, \frac{1}{p_{n}}\right) \stackrel{\mathrm{w}}{\succeq}\left(\frac{1}{p_{1}^{*}}, \ldots, \frac{1}{p_{n}^{*}}\right)
$$

implies that

$$
\sum_{i=j}^{n} \frac{1}{p_{i}} \leq \sum_{i=j}^{n} \frac{1}{p_{i}^{*}} \quad \text { for } j=1, \ldots, n
$$


If

$$
\frac{1}{p_{1}} \leq \frac{1}{p_{n}^{*}}
$$

then we have $1 / p_{i} \leq 1 / p_{i}^{*}$ for $i=1, \ldots, n$. Hence,

$$
X_{p_{i}} \geq \operatorname{lr} X_{p_{i}^{*}}, \quad i=1, \ldots, n .
$$

The likelihood ratio order has the preservation property that if $X \geq \operatorname{lr} Y$ and $Z$ is independent of $X$ and $Y$, where $X, Y$, and $Z$ have log-concave densities, then $X+Z \geq \operatorname{lr} Y+Z$ (see Theorem 1.C.9 of Shaked and Shanthikumar (2007)). Since Bernoulli random variables have log-concave densities, and the sum of independent random variables with log-concave densities has a log-concave density (see Theorem 2.18 of Dharmadhikari and Joag-Dev (1988)), it follows that

$$
\sum_{i=1}^{n} X_{p_{i}} \geq \operatorname{lr} \sum_{i=1}^{n} X_{p_{i}^{*}}
$$

Now, if

$$
\frac{1}{p_{1}}>\frac{1}{p_{n}^{*}}
$$

then there must exist some integer $k$ such that

$$
\frac{1}{p_{k+1}} \leq \frac{1}{p_{n}^{*}}<\frac{1}{p_{k}}
$$

Observing that

$$
\left(\frac{1}{p_{k}}, \frac{1}{p_{k+1}}\right) \stackrel{\mathrm{w}}{\succeq}\left(\frac{1}{p_{n}^{*}}, \frac{1}{p_{k}}+\frac{1}{p_{k+1}}-\frac{1}{p_{n}^{*}}\right),
$$

due to Lemma 3.2, we have

$$
X_{p_{k}}+X_{p_{k+1}} \geq \operatorname{lr} X_{p_{n}^{*}}+Z
$$

where $Z$ is a Bernoulli random variable with parameter $p=1 /\left\{1 / p_{k}+1 / p_{k+1}-1 / p_{n}^{*}\right\}$, independent of $X_{p_{i}^{*}}$ for $i=1, \ldots, n$.

Meanwhile, we have

$$
\left(\frac{1}{p_{1}}, \ldots, \frac{1}{p_{k-1}}, \frac{1}{p}, \frac{1}{p_{k+2}}, \ldots, \frac{1}{p_{n}}\right) \stackrel{\mathrm{w}}{\succeq}\left(\frac{1}{p_{1}^{*}}, \ldots, \frac{1}{p_{n-1}^{*}}\right) .
$$

By induction,

$$
X_{p_{1}}+\cdots+X_{p_{k-1}}+Z+X_{p_{k+2}}+\cdots+X_{p_{n}} \geq \operatorname{lr} X_{p_{1}^{*}}+\cdots+X_{p_{n-1}^{*}} .
$$

From (3.6) and Theorem 1.C.9 of Shaked and Shanthikumar (2007), it follows that

$$
X_{p_{1}}+\cdots+X_{p_{n}} \geq \operatorname{lr} X_{p_{1}}+\cdots+X_{p_{k-1}}+Z+X_{p_{n}^{*}}+X_{p_{k+2}}+\cdots+X_{p_{n}} .
$$

Hence, by (3.7) we have

$$
X_{p_{1}}+\cdots+X_{p_{k-1}}+Z+X_{p_{n}^{*}}+X_{p_{k+2}}+\cdots+X_{p_{n}} \geq \operatorname{lr} X_{p_{1}^{*}}+\cdots+X_{p_{n}^{*}} .
$$

Now, upon combining (3.8) and (3.9), the required result follows immediately. 
Using a similar argument and Lemma 3.3, we obtain the following result.

Theorem 3.2. Let $X_{p_{1}}, \ldots, X_{p_{n}}$ be independent Bernoulli random variables with parameters $p_{1}, \ldots, p_{2}$, respectively. Then,

$$
\begin{gathered}
\left(-\log \left(p_{1}\right), \ldots,-\log \left(p_{n}\right)\right) \stackrel{\mathrm{w}}{\succeq}\left(-\log \left(p_{1}^{*}\right), \ldots,-\log \left(p_{n}^{*}\right)\right) \\
\Longrightarrow X_{p_{1}}+\cdots+X_{p_{n}} \geq_{\mathrm{rh}} X_{p_{1}^{*}}+\cdots+X_{p_{n}^{*}}
\end{gathered}
$$

\section{Acknowledgement}

The authors are grateful to the anonymous referee for some constructive comments and suggestions which led to this improved version of the paper.

\section{References}

Boland, P. J. (2007). The probability distribution for the number of successes in independent trials. Commun. Statist. Theory Meth. 36, 1327-1331.

Boland, P. J. and Proschan, F. (1983). The reliability of $k$-out-of- $n$ systems. Ann. Prob. 11, 760-764.

Boland, P. J. AND SINGH, H. (2006). Stochastic comparisons of Bernoulli sums and binomial random variables. In Advances in Distribution Theory, Order Statistics, and Inference, eds N. Balakrishnan, E. Castillo and J. M. Sarabia, Birkhäuser, Boston, MA, pp. 3-11,

Boland, P. J., Singh, H. AND CUKIC, B. (2002). Stochastic orders in partition and random testing of software. J. Appl. Prob. 39, 555-565.

Boland, P. J., Singh, H. ANd Cukic, B. (2004). The stochastic precedence ordering with applications in sampling and testing. J. Appl. Prob. 41, 73-82.

Bon, J.-L. AND PǍLtǎnea, E. (1999). Ordering properties of convolutions of exponential random variables. Lifetime Data Anal. 5, 185-192.

Dharmadhikari, S. W. And Joag-Dev, K. (1988). Unimodality, Convexity, and Applications. Academic Press, Boston, MA.

Gleser, L. J. (1975). On the distribution of the number of successes in independent trials. Ann. Prob. 3, 182-188.

Hoeffoing, W. (1956). On the distribution of the number of successes in independent trials. Ann. Math. Statist. 27, 713-721.

Hu, T. And RuAn, L. (2004). A note on multivariate stochastic comparisons of Bernoulli random variables. J. Statist. Planning Infer. 126, 281-288.

Karlin, S. And NoviKoff, A. (1963). Generalized convex inequalities. Pacific J. Math. 13, 1251-1279.

Kochar, S. AND XU, M. (2011). The tail behavior of the convolutions of gamma random variables. J. Statist. Planning Infer. 141, 418-428.

MA, C. (2000). Convex orders for linear combinations of random variables. J. Statist. Planning Infer. 84, 11-25.

MaO, T., Hu, T. AND ZhaO, P. (2010). Ordering convolutions of heterogeneous exponential and geometric distribution revisited. Prob. Eng. Inf. Sci. 24, 329-348.

Marshall, A. AND OlKin, I. (1979). Inequalities: Theory of Majorization and Its Applications. Academic Press, New York.

Pledger, G. and Proschan, F. (1971). Comparisons of order statistics and of spacings from heterogeneous distributions. In Optimizing Methods in Statistics, Academic Press, New York, pp. 89-113.

Shaked, M. and Shanthikumar, J. G. (2007). Stochastic Orders and Their Applications. Springer, New York.

WANG, Y. H. (1993). On the number of successes in independent trials. Statistica Sinica 3, 295-312.

XU, M. AND BALAKRISHnan, N. (2010). On the convolution of heterogeneous Bernoulli random variables with applications. Tech. Rep., Illinois State University.

Zhao, P. and Balakrishnan, N. (2010). Ordering properties of convolutions of heterogeneous Erlang and Pascal random variables. Statist. Prob. Lett. 80, 969-974. 